\title{
Protective effect of edaravone on blood-brain barrier by affecting NRF-2/HO-1 signaling pathway
}

\author{
JING LIU, YAN JIANG, GUANGPING ZHANG, ZAIHONG LIN and SHU DU \\ Fourth Department of Neurology, The Third Affiliated Hospital of \\ Qiqihar Medical University, Qiqihar, Heilongjiang 161000, P.R. China
}

Received January 24, 2019; Accepted May 21, 2019

DOI: $10.3892 /$ etm.2019.7859

\begin{abstract}
Protective effect of edaravone on blood-brain barrier (BBB) in experimental cerebral infarction rats was investigated. SD rats were prepared as the permanent middle cerebral artery occlusion model and randomly divided into 4 groups: cerebral infarction model group, edaravone low, medium and high dose groups. Healthy rats only for operation and no filament were selected as the sham operation control group. Rats in the cerebral infarction model group and the control group were given normal saline, and those in the edaravone low, medium and high dose groups were given edaravone 10,15 and $20 \mathrm{mg} / \mathrm{kg}$, respectively. The survival status, the body weight and neurological function score before and after treatment, the brain water content and the permeability of the bloodbrain barrier after treatment were measured. The expression levels of NFE2-related factor 2 (NRF2) and hemeoxygenase 1 (HO-1) in rat brain tissue were detected by western blotting. Levels of peripheral blood malondialdehyde (MDA), superoxide dismutase (SOD) and glutathione (GSH) were detected by ELISA. The state of the rats in three edaravone groups was improved compared with that of the cerebral infarction group. Compared with the cerebral infarction model group, the body weight was significantly increased after treatment and the neurological function score, brain tissue water content and BBB permeability were significantly decreased in three edaravone groups $(\mathrm{P}<0.05)$.Compared with the model group of cerebral infarction, the expression of NRF-2 and HO-1 in the brain of the three edaravone groups was significantly higher $(\mathrm{P}<0.05)$. Compared with the model group of cerebral infarction, the expression of MDA and GSH in the three edaravone groups was significantly decreased, GSH and SOD was increased $(\mathrm{P}<0.05)$, in a dose-dependent manner. Edaravone might play a protective role in the $\mathrm{BBB}$ by activating the NRF-2/HO-1 signaling pathway.
\end{abstract}

Correspondence to: Dr Jing Liu, Fourth Department of Neurology, The Third Affiliated Hospital of Qiqihar Medical University, 27 Taishun Street, Qiqihar, Heilongjiang 161000, P.R. China E-mail: liujing830@126.com

Key words: edaravone, cerebral infarction, NFE2-related factor 2/ hemeoxygenase 1 signaling pathway, blood-brain barrier

\section{Introduction}

Cerebral infarction (CI) has the characteristics of long recovery time and unsatisfactory prognosis. The disease progresses gradually or stepwise, and is the main cause of human disability and death (1). In recent years, the number of people who died or became disabled from cerebral infarction has shown an upward trend.

Clinical treatment of cerebral infarction often uses thrombolysis technology. Reperfusion after thrombolysis is beneficial to the recovery of neurons in reversible damage in the ischemic area, leading to brain microvascular damage, blood-brain barrier (BBB) injury, and angiogenic brain edema, aggravating brain damage, which are also serious complications of thrombolysis, so protecting or reducing BBB injury is of great significance for the treatment of cerebral infarction. BBB is a special barrier between the blood circulation and nerve tissue of the brain. Its function is to maintain the homeostasis of the brain and regulate the balance of material exchange in the brain. BBB injury is the main cause of vasogenic cerebral edema and worsening of the disease in ischemic stroke, maintenance and improvement of BBB damage are important for the treatment of ischemic stroke and improvement of prognosis (2-4). The nuclear transcription factor NRF-2/hemeoxygenase 1 (NRF-2/HO-1) signaling pathway is the most important cell defense mechanism, and studies have shown that NRF-2/HO-1 signaling pathway activation can improve the integrity and stability of the BBB, and is a key target for the treatment of BBB in cerebral infarction $(5,6)$. Edaravone is a clinically used drug for the treatment and prevention of ischemic stroke. It inhibits delayed neuronal death, and inhibits the production of lipid peroxide, damage of vascular endothelial cells and ischemic cerebral edema and improves neurological function $(7,8)$, there are reports that edaravone also has effects in improving BBB, but the mechanism is still unclear $(9,10)$, and opinions vary. Niu et al (11) considered that edaravone inhibits the expression of occludin around the injured area of BBB, and Tsuruoka et al (12) that the expression of MMP-9 inhibited damaged brain tissue to protect the function of $\mathrm{BBB}$. However, the relationship between edaravone and NRF-2/HO-1 signaling pathway has rarely been reported.

The aim of the study was to investigate the effect of edaravone on the treatment of ischemic stroke with BBB injury and 
the influence on NRF-2/HO-1 signaling pathway, in order to provide a reference for the clinical treatment of ischemic stroke with edaravone.

\section{Materials and methods}

Test animals. Eighty adult healthy male SD rats, with body weight of 200-250 g, were provided by Hunan Weitong Lihua Experimental Animal Co., Ltd., animal certificate number: SCXK (Hunan) 2009-0004. After 1 week of adaptive feeding, the experiment was carried out. During the feeding period, the rats were given free access to water, with constant temperature and humidity, and artificial lighting for $12 \mathrm{~h}$ each day. All the operations strictly abided by the ethical rules for experimental animals.

The study was approved by the Ethics Committee of The Third Affiliated Hospital of Qiqihar Medical University (Qiqihar, China).

Permanent cerebral artery occlusion model and evaluation. A modified Longa method was used to make a permanent middle cerebral artery occlusion model (cerebral infarction rats): after abdominal anesthesia, rats were fixed on the operating table in supine position, with preserved skin sterilized. Then $1 \mathrm{~cm}$ median incision of the neck was taken to bluntly separate the neck, and the right common carotid artery (CCA), external carotid artery (ECA) and internal carotid artery (ICA)were separated, ECA trunk was separated, ligated $2 \mathrm{~mm}$ from the CCA bifurcation and separated, continuing to separate from the ICA to the base of the skull, the ICA was exposed, CCA was temporarily clamped, a small opening was cut in the ECA stump, the suture was inserted into the ICA through the ECA stump, when slight resistance of the incoming suture was felt, the end of the filament suture was ligated and cut, CCA blood flow was restored, and wounds were sutured. After successful model preparation, the rats were sacrificed at $24 \mathrm{~h}$ after surgery for neurological function scores, and $\geq 2$ were selected as study subjects. Neurological function score: 0 point, no neurological deficit; 1 point, the left forelimb of the rat was difficult to fully extend; 2 points, left front limb flexion; 3 points, slightly to the left when walking; 4 points, severe left side turn; 5 points, rats fell to the left while walking.

Grouping and administration. Eighty rats with cerebral infarction were randomly divided into 4 groups: cerebral infarction model group, edaravone low, medium and high dose groups. Healthy rats were taken from the same batch as sham operation group, the rat model was established in the same way as the 'permanent middle cerebral artery occlusion model', but only the vascular was separated and the filament suture was not inserted ( $\mathrm{n}=20$ per group). Rats in the cerebral infarction model group and the control group were given normal saline by intragastric administration. The edaravone low, medium and high dose groups were intragastrically administered with 10,15 and $20 \mathrm{mg} / \mathrm{kg}$, respectively, for 14 days.

\section{Indicator detection}

Survival status. The survival status of all the groups of rats was recorded, including activity, fur and diet.
Body mass and neurological function scores. The body mass and neurological function scores of all the groups of rats before and after administration were recorded. The neurological function score was the same as above.

Brain tissue water content. After treatment, 10 rats in each group were sacrificed, and the brain tissue of the first half of the hematoma center was taken, weighed and placed in an oven at about $100^{\circ} \mathrm{C}$ for $24 \mathrm{~h}$ and the dry weight was recorded. The water content of brain tissue was calculated as follows: brain tissue water content $(\%)=($ fresh mass - dry mass $) /$ fresh mass $100 \%$.

Blood-brain barrier permeability. The Evans blue formamide (EB) method was used. After treatment, the remaining 10 rats in each group were taken. After general anesthesia, $2 \mathrm{ml} / \mathrm{kg}$ of $2 \% \mathrm{~EB}$ was injected through the tail vein. The thoracic left ventricle was perfused with normal saline at a pressure of $110 \mathrm{mmHg}$ until the liquid flowing out of the right atrium was colorless. The brain was taken by decapitation, and the inner part of the hematoma was weighed and placed in formamide, $1 \mathrm{ml} / 100 \mathrm{~g}$ of brain tissue, and incubated at $60^{\circ} \mathrm{C}$ overnight. After centrifugation at $300 \mathrm{xg}$ and $4^{\circ} \mathrm{C}$ for 10 min with a radius of $8 \mathrm{~cm}$, the OD value of the supernatant was determined by UV751GD ultraviolet spectrophotometer (Shanghai Precision Scientific Instrument Co., Ltd.) at a wavelength of $632 \mathrm{~nm}$, and the EB level was calculated. The higher the EB, the higher the blood-brain barrier permeability.

Western blot analysis of NRF-2 and HO-1 protein expression. The total protein in remaining hematoma brain tissue was extracted by RIPA buffer, and was detected by BCA method. Then, $10 \%$ SDS-PAGE was performed and transferred to a PVDF membrane and $30 \mu \mathrm{g}$ of protein was loaded per lane. The cells were blocked with $5 \%$ skim milk powder at $4{ }^{\circ} \mathrm{C}$ for $1 \mathrm{~h}$. NRF-2 $(1: 250)$ and HO-1 $(1: 2,000)$ primary antibodies were added dropwise and incubated overnight. The antibodies were incubated again and visualized with horseradish enzymelabeled secondary antibody $(1: 2,000)$ prior to scanning by a Bio-Rad 2000 gel imaging system. Absorbance values were analyzed using the Image-Pro Plus 6.0 Quantityone software. NRF-2 (bs-1074R-1) and HO-1 (bs-0827R-1) were rabbit anti-human antibodies and were from Shanghai Hengfei Biotechnology Co., Ltd. $\beta$-actin (item no.: SC.1616, Santa Cruz, Biotechnology, Inc.) was the internal reference.

Oxidative stress index. After treatment of rats in all the groups, blood was taken from ocular venous plexus prior to euthanasia, and the serum was obtained via centrifugation at 4,000 $\mathrm{x} g$ at $25^{\circ} \mathrm{C}$ for $5 \mathrm{~min}$. ELISA method was performed and the OD values of malonyldialdehyde (MDA), superoxide dismutase (SOD) and glutathione (GSH) in serum was read by MRX Revelation type microplate reader (Dynex) according to the kit instructions, the expression levels were determined by the standard curve of each index. MDA, SOD and GSH kits were purchased from Nanjing Jiancheng Bioengineering Co., Ltd.

Statistical analysis. The data were processed by SPSS statistical software package (SPSS, Inc.). Measurement data, such as NRF-2 and HO-1 protein relative expression, were expressed 
A

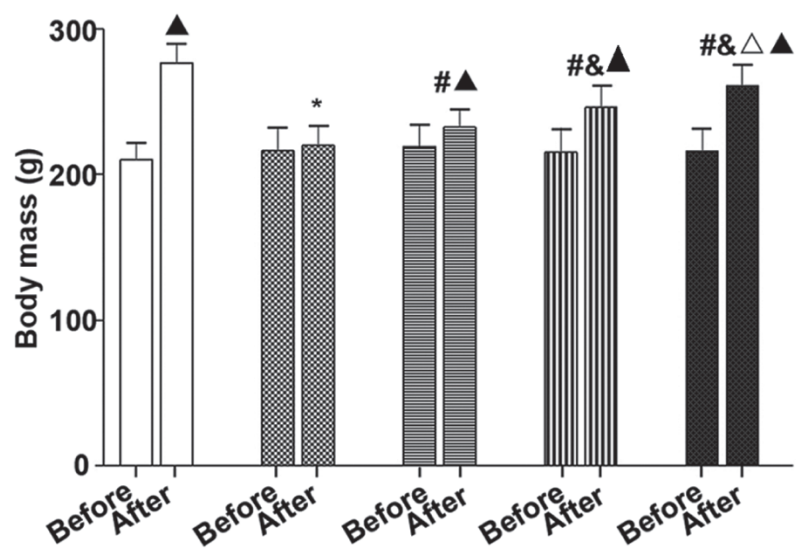

B

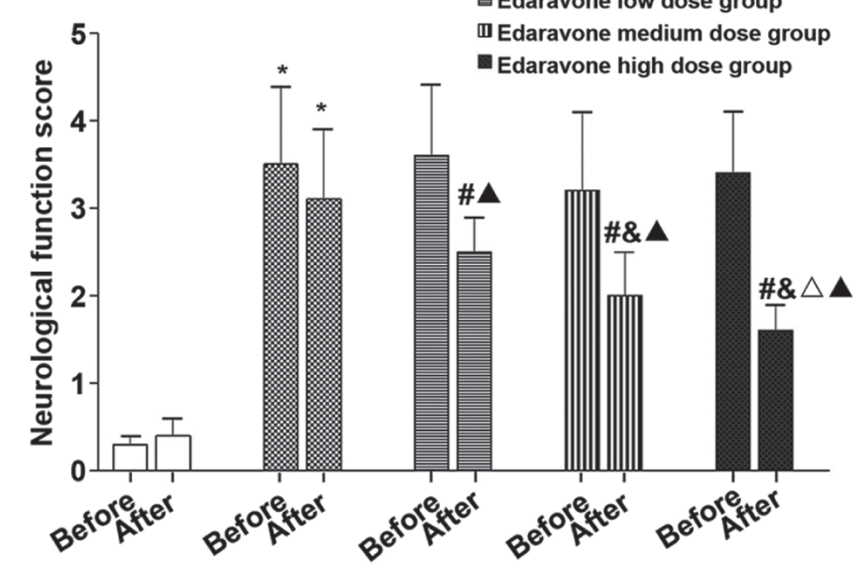

Figure 1. Comparison of body mass and neurological function scores before and after treatment in five groups of rats (n=20). (A) Body mass, (B) neurological function score; comparing cerebral infarction model group with control group, ${ }^{*} \mathrm{P}<0.05$; compared with cerebral infarction model group, ${ }^{\#} \mathrm{P}<0.05$; compared with edaravone low dose group, ${ }^{\&} \mathrm{P}<0.05$; compared with the edaravone low dose group, ${ }^{\triangle} \mathrm{P}<0.05$; compared with the edaravone medium dose group, ${ }^{\triangle} \mathrm{P}<0.05$; compared with before treatment, ${ }^{\wedge} \mathrm{P}<0.05$. Before: before treatment, After: after treatment.

A

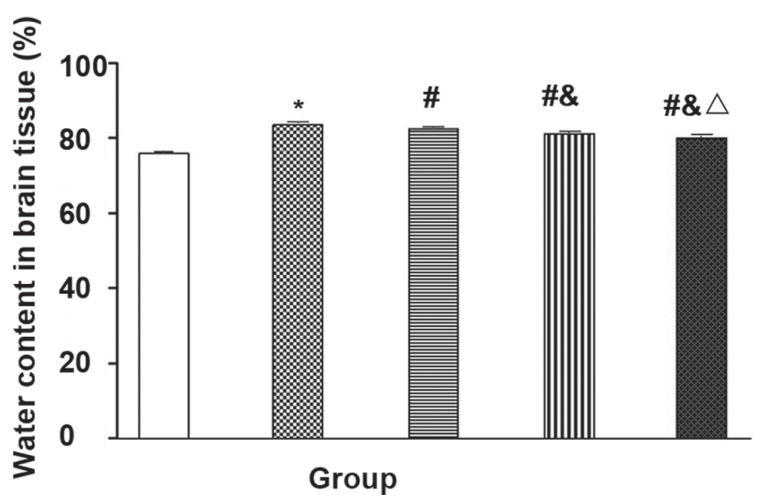

B

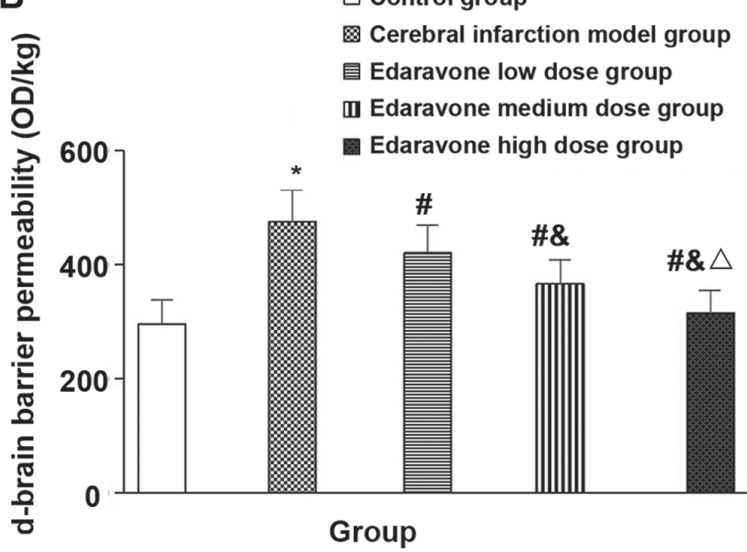

Figure 2. Comparison of brain tissue water content and blood-brain barrier permeability in five groups of rats. (A) Brain tissue water content, (B) blood-brain barrier permeability; cerebral infarction model group compared with control group, ${ }^{*} \mathrm{P}<0.05$; compared with cerebral infarction model group, ${ }^{\#} \mathrm{P}<0.05$; compared with edaravone low dose group, ${ }^{\&} \mathrm{P}<0.05$; compared with the edaravone medium dose group, ${ }^{\triangle} \mathrm{P}<0.05$.

as mean standard deviation (mean $\pm \mathrm{SD}$ ), and intra-group comparison was performed using variance analysis. The SNQ-q test was used for pairwise comparison between groups, $\alpha=0.05$ for the test level. $\mathrm{P}<0.05$ was considered to indicate a statistically significant difference.

\section{Results}

Survival status of the five groups of rats. The rats in the sham operation group were active, the diet was normal, the hair color was regular, sensitive to external stimuli, the activity of rats with cerebral infarction was significantly reduced, the diet was significantly reduced, the hair color of the rats was poor, and the spirit and aggression both were poor, and the state of the edaravone rats was improved to a different extent than that of cerebral infarction rats.

Comparison of the body weight, neurological function score, brain tissue water content and blood-brain barrier permeability of the five groups of rats. Compared with the control group, the body mass of the cerebral infarction model group was significantly reduced after treatment $(\mathrm{t}=13.937, \mathrm{P}<0.001)$, neurological function score $(\mathrm{t}=14.623$, $\mathrm{P}<0.001)$, brain tissue water content $(\mathrm{t}=19.711, \mathrm{P}<0.001)$ and blood-brain barrier permeability $(\mathrm{t}=26.068, \mathrm{P}<0.001)$ were significantly increased. Compared with the cerebral infarction model group, the body weight of the edaravone group was significantly increased after treatment $(\mathrm{F}=31.122, \mathrm{P}<0.001)$, neurological function score $(\mathrm{F}=29.474, \mathrm{P}<0.001)$, brain tissue water content $(\mathrm{F}=60.185, \mathrm{P}<0.001)$ and blood-brain barrier permeability $(\mathrm{F}=22.986, \mathrm{P}<0.001)$ were significantly reduced, and showed a dose-dependent trend (Figs. 1 and 2).

Expression of NRF-2 and HO-1 in the brain of five groups of rats. Compared with the control group, the expression of NRF-2 $(\mathrm{t}=2.460, \mathrm{P}=0.036)$ and HO-1 $(\mathrm{t}=2.683, \mathrm{P}=0.025)$ in the brain infarction model was significantly increased. Compared with the cerebral infarction model group, the expression of 
A

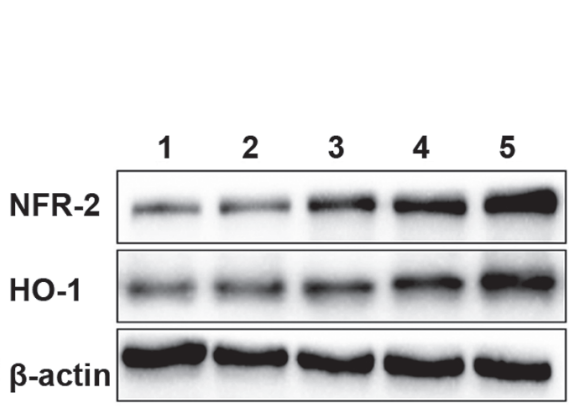

B

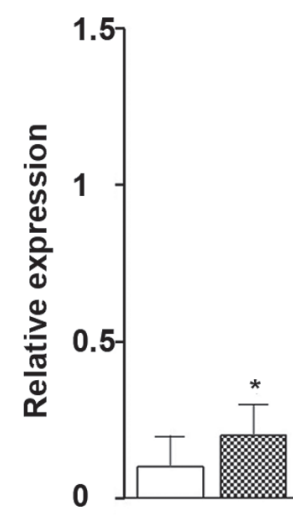

$\square$ Control group

Cerebral infarction model group

目Edaravone low dose group

mEdaravone medium dose group

\#\& $\triangle$ Edaravone high dose group

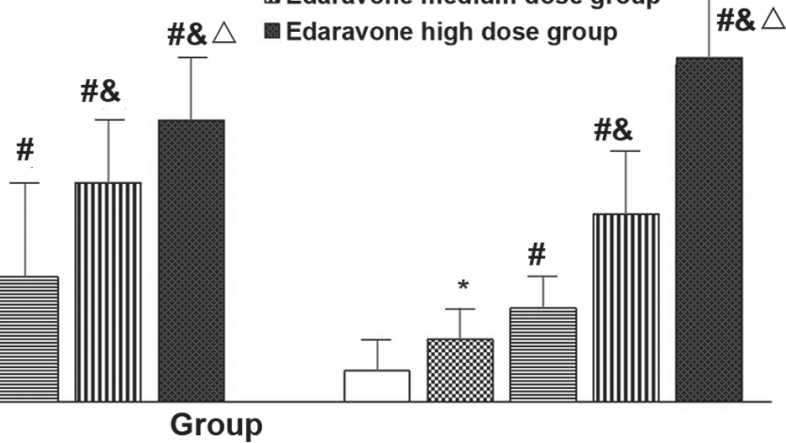

Figure 3. Expression of NRF-2 and HO-1 in the brains of five groups of rats (western blot, n=10). Lane 1, control group; 2, cerebral infarction model group; 3 , edaravone low dose group; 4, edaravone medium dose group; 5, edaravone high dose group. (A) Western blot result of NRF-2 and HO-1 protein. (B) Quantitative analysis of NRF-2 and HO-1 protein. The brain infarction model group compared with the control group, "P<0.05; compared with the cerebral infarction model group, ${ }^{\#} \mathrm{P}<0.05$; compared with the edaravone low dose group, ${ }^{\circledR} \mathrm{P}<0.05$; compared with the edaravone medium dose group, ${ }^{\wedge} \mathrm{P}<0.05$.

A
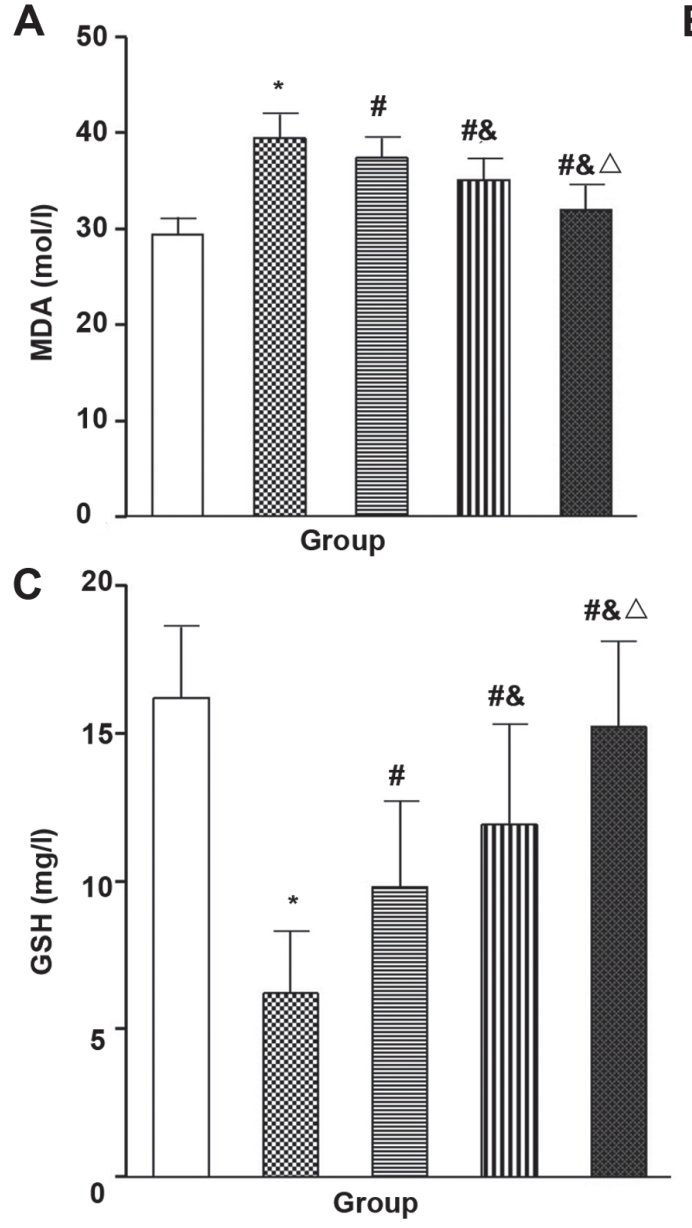

B

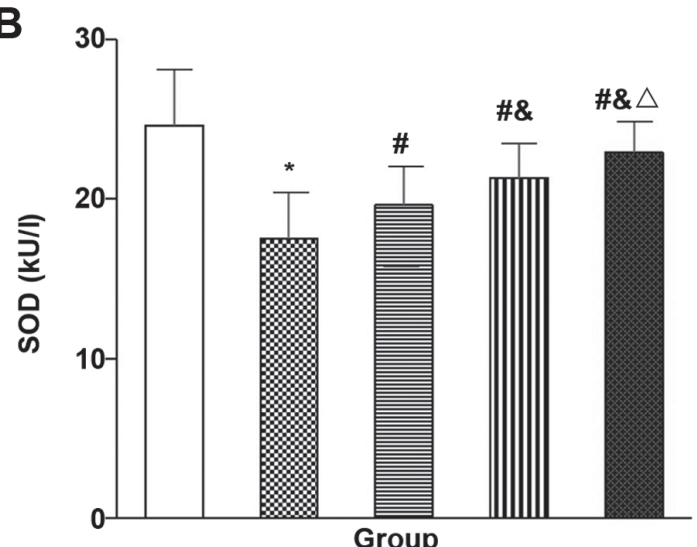

Group

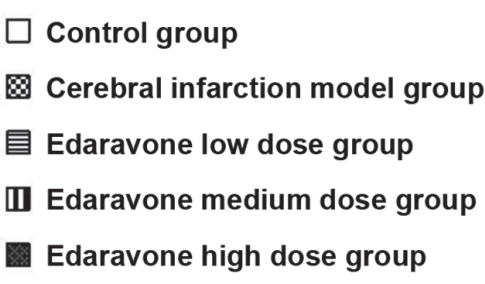

$\square$ Control group

Cerebral infarction model group

dose group

Il Edaravone medium dose group

Edaravone high dose group

Figure 4. Comparison of oxidative stress in peripheral blood of five groups of rats $(n=10)$. (A) MDA level in peripheral blood of five groups of rats. (B) SOD level in peripheral blood of five groups of rats. (C) GSH level in peripheral blood of five groups of rats. Compared with the control group, ${ }^{\mathrm{P}} \mathrm{P}<0.05 ;$ compared with the cerebral infarction model group, ${ }^{\#} \mathrm{P}<0.05$; compared with the edaravone low dose group, ${ }^{\&} \mathrm{P}<0.05$; compared with the edaravone medium dose group, ${ }^{\wedge} \mathrm{P}<0.05$.

NRF-2 $(\mathrm{F}=21.481, \mathrm{P}<0.001)$ and HO-1 $(\mathrm{F}=65.333, \mathrm{P}<0.001)$ in the edaravone group was significantly increased and in a dose-dependent manner (Fig. 3).
Comparison of oxidative stress indicators in the peripheral blood of five groups of rats. Compared with the control group, the MDA $(\mathrm{t}=10.646, \mathrm{P}<0.001)$ in the cerebral infarction model 
group were significantly increased, and the GSH $(\mathrm{t}=49.40$, $\mathrm{P}=0.001)$ and $\mathrm{SOD}(\mathrm{t}=9.916, \mathrm{P}<0.001)$ were significantly decreased, and compared with the brain infarction model group, the MDA ( $\mathrm{F}=19.051, \mathrm{P}<0.001)$ was significantly lower in the edaravone group, and the GSH $(\mathrm{F}=9.667, \mathrm{P}<0.001)$ and SOD $(\mathrm{t}=17.374, \mathrm{P}<0.001)$ were significantly increased, with a dose-dependence trend (Fig. 4).

\section{Discussion}

Cerebral edema after cerebral infarction is vasogenic cerebral edema caused by impaired BBB, increased permeability and plasma exudation, and the cells secrete a large number of reactive oxygen species (ROS), which can participate in oxidative stress and the release of cytotoxic substances, as shown in the results of this study, the survival state of rats with cerebral infarction decreased, the brain water content increased, while the MDA of peripheral blood with oxidative stress were significantly increased, and the antioxidant GSH and SOD were significantly reduced.

The NRF2/HO-1 pathway is an important antioxidant and cytotoxic defense mechanism in cells, which is of great significance in stabilizing and protecting the integrity of the blood-brain barrier. Under physiological conditions, the molecular chaperone Kelch-like epichlorohydrin-related protein-1 (Keapl) is coupled with NRF2, binds to actin, rapidly degraded by ubiquitination, and has a short half-life. Under oxidative stress conditions such as ischemia and hypoxia, NRF2 is decoupled from Keap, and a large number of NRF2s are activated to enter the nucleus and initiate NRF2 downstream target gene expression. As a brain protective factor, NRF-2, through the binding of cell stress signaling proteins, participates in the transcription process, and then regulates the expression of various detoxification enzymes and antioxidant enzymes downstream, which can regulate oxidative stress after cerebral infarction (13). HO-1 is an important downstream target gene of NRF2. HO-1 catalyzes the degradation of heme, and the products of degradation have anti-oxidative damage function. It has anti-inflammatory, anti-apoptotic and antiproliferative synergistic effects on HO-1. It plays an important protective role in various diseases.

A large number of studies have shown that activation of the NRF2/HO-1 pathway and induction of high expression of NRF2 and/or HO-1 may play an important role in antioxidation, anti-apoptosis and protection of the blood-brain barrier (14). The results of this study showed that compared with the control group, the expression of NRF2 and HO-1 protein in the brain of rats with cerebral infarction increased, suggesting that the NRF2/HO-1 pathway is activated, which is the endogenous and adaptive protection mechanism of the body to initiate cerebral infarction, but this compensatory ability is relatively limited, no drug intervention, no improvement in neurological function scores in rats, suggesting that nerve function may be lost, brain tissue damage may occur, with the administration of edaravone and increased dose of edaravone, the expression of NRF2 and HO-1 protein in the brain increase in a dose-dependent manner, edaravone may promote the decoupling of NRF2 from the cytoplasmic chaperone Keap through the pathway of inhibiting apoptosis, transport to the nucleus, initiate the relative expression of the downstream gene HO-1 of NRF2, promote the body to exert stronger anti-oxidative stress, improve the integrity of BBB, and then protect the cranial nerves of infarcted rats (15). This result is similar to that reported in the literature. For example, studies of Liu et al (16) have shown that edaravone protects the hippocampal neurons of kainic acid-induced epileptic rats by activating Nrf2/HO-1 channels; Zhang et al (17) considered that edaravone reduces neuronal apoptosis in the hippocampus by activating the Nrf2/HO-1 signaling pathway, which in turn produces neuroprotection in a mouse model of pneumococcal meningitis. Cechetti et al (18) reported that edaravone reduces oxidative stress induced by chronic cerebral hypoperfusion injury and is associated with activation of $\mathrm{Nrf} 2 / \mathrm{HO}-1$ signaling pathway.

There is insufficient research on this topic in vivo, although the administration of edaravone in rats with cerebral infarction reduces the permeability of blood-brain barrier and brain edema, increases the expression of Nrf2 and HO-1 protein in brain tissue, whether the upregulation of Nrf2 and HO-1 protein expression is a direct or indirect effect of edaravone is not clear, and further studies should be combined with ex vivo analysis.

In summary, edaravone may exert a blood-brain barrier protection by activating the NRF-2/HO-1 signaling pathway.

\section{Acknowledgements}

Not applicable.

\section{Funding}

This study was supported by the Qiqihar Science and Technology Plan Project (SFGG-201512).

\section{Availability of data and materials}

The datasets used and/or analyzed during the present study are available from the corresponding author on reasonable request.

\section{Authors' contributions}

JL wrote the manuscript. JL and YJ were responsible for the cerebral artery occlusion model construction. GZ and ZL performed western blotting. SD was responsible for the data analysis and interpretation. All authors read and approved the final manuscript.

\section{Ethics approval and consent to participate}

The study was approved by the Ethics Committee of The Third Affiliated Hospital of Qiqihar Medical University (Qiqihar, China).

\section{Patient consent for publication}

Not applicable.

\section{Competing interests}

The authors declare that they have no competing interests. 


\section{References}

1. Nagai N, Yoshioka C, Ito Y, Funakami Y, Nishikawa H and Kawabata A: Intravenous administration of cilostazol nanoparticles ameliorates acute ischemic stroke in a cerebral ischemia/ reperfusion-induced injury model. Int J Mol Sci 16: 29329-29344, 2015.

2. Chen S, Chen Z, Cui J, McCrary ML, Song H, Mobashery S, Chang $\mathrm{M}$ and $\mathrm{Gu} \mathrm{Z}$ : Early abrogation of gelatinase activity extends the time window for tPA thrombolysis after embolic focal cerebral ischemia in mice. eNeuro 5: pii: ENEURO.0391-17.2018, 2018.

3. Li M, Wen Y, Zhang R, Xie F, Zhang G and Qin X: Adenoviral vector-induced silencing of RGMa attenuates blood-brain barrier dysfunction in a rat model of $\mathrm{MCAO} /$ reperfusion. Brain Res Bull 142: 54-62, 2018.

4. Liu X, Kiss GK, Mellender SJ, Weiss HR and Chi OZ: Activation of Akt by SC79 decreased cerebral infarct in early cerebral ischemia-reperfusion despite increased BBB disruption. Neurosci Lett 681: 78-82, 2018.

5. Liu C, Zhong L, Tian XL and Han YC: Protective effects of 8-MOP on blood-brain barrier via the $\mathrm{Nrf}-2 / \mathrm{HO}-1$ pathway in mice model of cerebral infarction. Eur Rev Med Pharmacol Sci 22: 4278-4287, 2018.

6. Liu Z, Ma C, Shen J, Wang D, Hao J and Hu Z: SDF-1/CXCR4 axis induces apoptosis of human degenerative nucleus pulposus cells via the NF-кB pathway. Mol Med Rep 14: 783-789, 2016.

7. Yamada H, Kikuchi R, Nakamura A and Miyazaki H: Severe reversible cerebral vasoconstriction syndrome with large posterior cerebral infarction. J Stroke Cerebrovasc Dis 27: 3043-3045, 2018.

8. Ohta Y, Takamatsu K, Fukushima T, Ikegami S, Takeda I, Ota T, Goto K and Abe K: Efficacy of the free radical scavenger, edaravone, for motor palsy of acute lacunar infarction. Intern Med 48: 593-596, 2009.

9. Chen Y and Zhao Y: Curative efficacy of penehyclidine combined with edaravone on acute cerebral infarction and their effects on serum TNF- $\alpha$ and NDS score in rats. Eur Rev Med Pharmacol Sci 22: 223-228, 2018.

10. Tóth AE, Walter FR, Bocsik A, Sántha P, Veszelka S, Nagy L, Puskás LG, Couraud PO, Takata F, Dohgu S, et al: Edaravone protects against methylglyoxal-induced barrier damage in human brain endothelial cells. PLoS One 9: e100152, 2014
11. Niu F, Song XY, Hu JF, Zuo W, Kong LL, Wang XF, Han N and Chen NH: IMM-H004, A new coumarin derivative, improved focal cerebral ischemia via blood-brain barrier protection in rats. J Stroke Cerebrovasc Dis 26: 2065-2073, 2017.

12. Tsuruoka A, Atsumi C, Mizukami H, Imai T, Hagiwara $Y$ and Hasegawa Y: Effects of edaravone, a free radical scavenger, on circulating levels of MMP-9 and hemorrhagic transformation in patients with intravenous thrombolysis using low-dose alteplase. J Stroke Cerebrovasc Dis 23: 2894-2899, 2014.

13. Li C, Wang R, Hu C, Wang H, Ma Q, Chen S and He Y: Pyridoxine exerts antioxidant effects in cell model of Alzheimer's disease via the Nrf-2/HO-1 pathway. Cell Mol Biol 64: 119-124, 2018.

14. Wang W, Wang X, Zhang XS and Liang CZ: Cryptotanshinone attenuates oxidative stress and inflammation through the regulation of Nrf-2 and $\mathrm{NF}-\kappa \mathrm{B}$ in mice with unilateral ureteral obstruction. Basic Clin Pharmacol Toxicol 123: 714-720, 2018.

15. Liu X, Zhang X, Ma K, Zhang R, Hou P, Sun B, Yuan S, Wang Z and Liu Z: Matrine alleviates early brain injury after experimental subarachnoid hemorrhage in rats: Possible involvement of PI3K/Akt-mediated NF- $\kappa$ B inhibition and Keap1/Nrf2dependent HO-1 inductionn. Cell Mol Biol (Noisy-le-grand) 62: 38-44, 2016.

16. Liu Z, Yang C, Meng X, Li Z, Lv C and Cao P: Neuroprotection of edaravone on the hippocampus of kainate-induced epilepsy rats through Nrf2/HO-1 pathway. Neurochem Int 112: 159-165, 2018.

17. Zhang D, Xiao Y,Lv P, Teng Z, Dong Y, Qi Q and Liu Z: Edaravone attenuates oxidative stress induced by chronic cerebral hypoperfusion injury: Role of ERK/Nrf2/HO-1 signaling pathway. Neurol Res 40: 1-10, 2018.

18. Cechetti F, Worm PV, Elsner VR, Bertoldi K, Sanches E, Ben J, Siqueira IR and Netto CA: Forced treadmill exercise prevents oxidative stress and memory deficits following chronic cerebral hypoperfusion in the rat. Neurobiol Learn Mem 97: 90-96, 2012. International (CC BY-NC-ND 4.0) License. 